\title{
The Childhood Asthma Management Program (CAMP): contributions to the understanding of therapy and the natural history of childhood asthma
}

\author{
Ronina A. Covar • Anne L. Fuhlbrigge • Paul Williams • \\ H. William Kelly • \\ for the Childhood Asthma Management Program \\ Research Group
}

Published online: 18 September 2012

(C) Springer Science+Business Media, LLC 2012

\begin{abstract}
The Childhood Asthma Management Program (CAMP) has been in continuous existence for almost two decades, which makes it the largest randomized, placebocontrolled clinical trial with extended follow-up for children with mild to moderate asthma. As such, its cumulative data from baseline, active treatment, and posttreatment have proved to be an invaluable resource for not only assessing the efficacy and safety of long-term inhaled corticosteroid therapy in childhood, but for discovery of many other aspects of childhood asthma, including genetics and biomarkers.
\end{abstract}

\section{R. A. Covar $(\bowtie)$}

National Jewish Health,

1400 Jackson Street,

Denver, CO 80206, USA

e-mail: covarr@njhealth.org

\author{
A. L. Fuhlbrigge \\ Pulmonary and Critical Care Division, Channing Division of \\ Network Medicine, Brigham and Women's Hospital, \\ Harvard Medical School, \\ 75 Francis Street, \\ Boston, MA, USA \\ e-mail: afuhlbrigge@partners.org \\ P. Williams \\ University of Washington School of Medicine, ASTHMA, \\ Inc Clinical Research Center, \\ 9725 Third Ave NE, Suite 500, \\ Seattle, WA 98115, USA \\ e-mail: pwilliams@nwasthma.com \\ H. W. Kelly \\ University of New Mexico Health Sciences Center, \\ 9828 Guadalupe Trail NW, \\ Albuquerque, NM 87114-2009, USA \\ e-mail: hwkelly@salud.unm.edu
}

Keywords Asthma $\cdot$ Childhood · Spirometry · Airway hyperresponsiveness $\cdot$ Inhaled corticosteroids .

Bronchodilators $\cdot$ Bronchodilator response $\cdot$ Allergy .

Pharmacogenomics $\cdot$ Bone mineral density $\cdot$ Vitamin D .

Linear growth $\cdot$ Cataracts $\cdot$ Hypothalamic-pituitary-adrenal axis $\cdot$ Remission $\cdot$ Progression $\cdot$ Exacerbation $\cdot$ Oral corticosteroids $\cdot$ Nedocromil $\cdot$ Forced expiratory volume in one second $\left(\mathrm{FEV}_{1}\right) \cdot$ Forced vital capacity $(\mathrm{FVC})$. Symptoms · Health care utilization · Phenotype $\cdot$ Genetic polymorphism $\cdot$ Eosinophils

\section{Introduction}

In the early 1990s, the Childhood Asthma Management Program (CAMP) was developed to determine whether long-term anti-inflammatory therapy could alter the natural history of mild to moderate asthma of childhood by improving lung growth as measured by post-bronchodilator forced expiratory volume in one second $\left(\mathrm{FEV}_{1}\right)$. The rationale for CAMP and study design have been extensively reviewed elsewhere [1,2]. Briefly, 1,041 children 5-12 years of age with mild-to-moderate persistent asthma were enrolled from December 1993 to September 1995 at eight clinical centers. After a 2-4 month screening and baseline period to establish the severity of asthma and the ability of the patients and parents to adhere to the complexity of the trial, patients were randomized to budesonide dry-powder inhaler $(200 \mu \mathrm{g}$ twice daily), nedocromil metered-dose inhaler ( $8 \mathrm{mg}$ twice daily), or matching placebos for 4-6 years (mean 4.3 years) (Fig. 1). The extensive baseline assessment including lung function, airway reactivity, height, weight, race, ethnicity, Tanner staging, bone mineral density (BMD), asthma 
history, allergen skin testing, dust sampling in the home, social economic status determination, and psychological and neurocognitive development testing provided one of the best characterized childhood asthma populations that would enable assessment of potential predictors of response to therapy. In addition, early genotyping of the children and their families, funded through an ancillary genetics study would enable assessment of genetic determinants of asthma, their interaction with phenotypic factors known to affect response, and analysis of the natural progression of the disease.

After the initial clinical trial, the patients were removed from randomized therapy and then followed for an additional 13 years (Fig. 1) in three successive continuation studies, to determine the effect of early treatment, and progression of the asthma into adulthood. During this period the patients were assessed by the CAMP staff but treated by their primary care physicians with recommendations sent by the CAMP physicians for asthma treatment based upon the National Institutes of Health guidelines for management of asthma [3]. The percentage of time subjects were maintained on inhaled corticosteroids (ICS) during the observation phase of the study was similar across the three treatment groups of the randomized clinical trial (budesonide (31.3\%), nedocromil (30.9\%), and placebo (30.2\%)). Being the longest and largest prospective study of childhood asthma, CAMP provided a unique opportunity to evaluate not just the outcomes of therapy but the natural history of the disease. The CAMP investigators have published over 140 papers featuring the data obtained from baseline, the treatment trial, and many years of follow-up. It is the purpose of this review to briefly emphasize the most clinically significant findings from CAMP to help clinicians in the care of childhood asthma. The long-term outcomes from both therapy, the natural history of the disease, and the phenotypic and genotypic predictors of responses will also be emphasized.

\section{Outcome of long-term therapy}

\section{Efficacy of anti-inflammatory therapy}

At baseline, children were (mean $\pm \mathrm{SD}$ ) $9.0 \pm 2.1$ years old, $2 / 3$ white, and $60 \%$ male. The participants had features of mild or moderate persistent asthma: mean $\pm \mathrm{SD}$ episode-free days of $10 \pm 8$ per month, $0.9 \pm 1.6$ night awakenings/month, use of $10 \pm 10$ puffs of albuterol/week, and prebronchodilator $\mathrm{FEV}_{1} \%$ predicted of $94 \pm 14$ (post-bronchodilator FEV1 \% predicted 103 \pm 13 ). Participants had moderate to severe degree of airway hyperresponsiveness (AHR: geometric mean $\mathrm{FEV}_{1} \mathrm{PC}_{20} 1.1 \pm 3.3 \mathrm{mg} \mathrm{mL}^{-1}$ ) [2].
Neither ICS nor nedocromil therapy resulted in a significant improvement in the primary outcome of postbronchodilator $\mathrm{FEV}_{1} \%$ predicted versus placebo $[2,4]$. However, compared with placebo, ICS treatment was associated with a greater improvement in pre-bronchodilator $\mathrm{FEV}_{1} \%$ predicted, AHR, and bronchodilator response (BDR), and with $43 \%$ less hospitalization, $45 \%$ fewer urgent care visits, and $43 \%$ fewer prednisone courses. Nedocromil, however, resulted in $27 \%$ fewer urgent care visits and $16 \%$ fewer prednisone courses. The following were also better in children treated with ICS compared to those treated with placebo: symptom score, albuterol rescue use, episode-free days, and longer time to first course of prednisone, and initiation of supplemental therapy with other controller. ICS therapy was also associated with a significant improvement in the total score on the children's depression inventory (i.e., indicating less depression) compared with placebo [2].

The first post-trial follow up study of $4.8 \pm 0.5$ years made the following key observations [5••]. There were no differences in lung function or AHR for participants treated with ICS and those on placebo in the original trial. During this follow up period, the participants incurred low rates of exacerbation; children initially treated with budesonide had $29 \%$ fewer prednisone courses $(P=0.05)$, and $36 \%$ fewer urgent care visits $(p=0.05)$ compared with those assigned placebo. However, an estimated 15 and 32 participants would need to be treated with ICS regularly for four years to prevent one prednisone course per year and one urgent care visit per year, respectively, after discontinuation of the treatment.

Inhaled anti-inflammatory therapy, specifically corticosteroids, and to a lesser extent, nedocromil, afforded advantages over placebo in different measures of asthma control during the trial; however, such differences in benefits between original treatments were not sustained after those were discontinued.

Potential adverse effects of corticosteroids in childhood asthma

Systemic corticosteroids are well known to produce a variety of adverse systemic effects when taken either continuously or frequently over many years including: reduced linear growth; reduced BMD; reduced hypothalamic-pituitary-adrenal (HPA) axis activity; and posterior subcapsular cataracts (PSCs) [6]. The CAMP trial provided a unique opportunity to evaluate the potential long-term systemic consequences of medium-dose ICS therapy in a prospective manner. In the initial trial a significant reduction in linear growth velocity was noted in the budesonide group; this occurred over the first two years and then returned to match that of the nedocromil and placebo groups [2]. The decrease in velocity resulted in a significant but small reduction in 
Fig. 1 Schematic diagram of the childhood asthma management program. 1,041 children aged 5 to 12 years with mild to moderate persistent asthma were randomized to receive budesonide, nedocromil, or placebo. After 4 to 6 years of treatment, the cohort participated in an observational study to characterize the natural history of childhood asthma

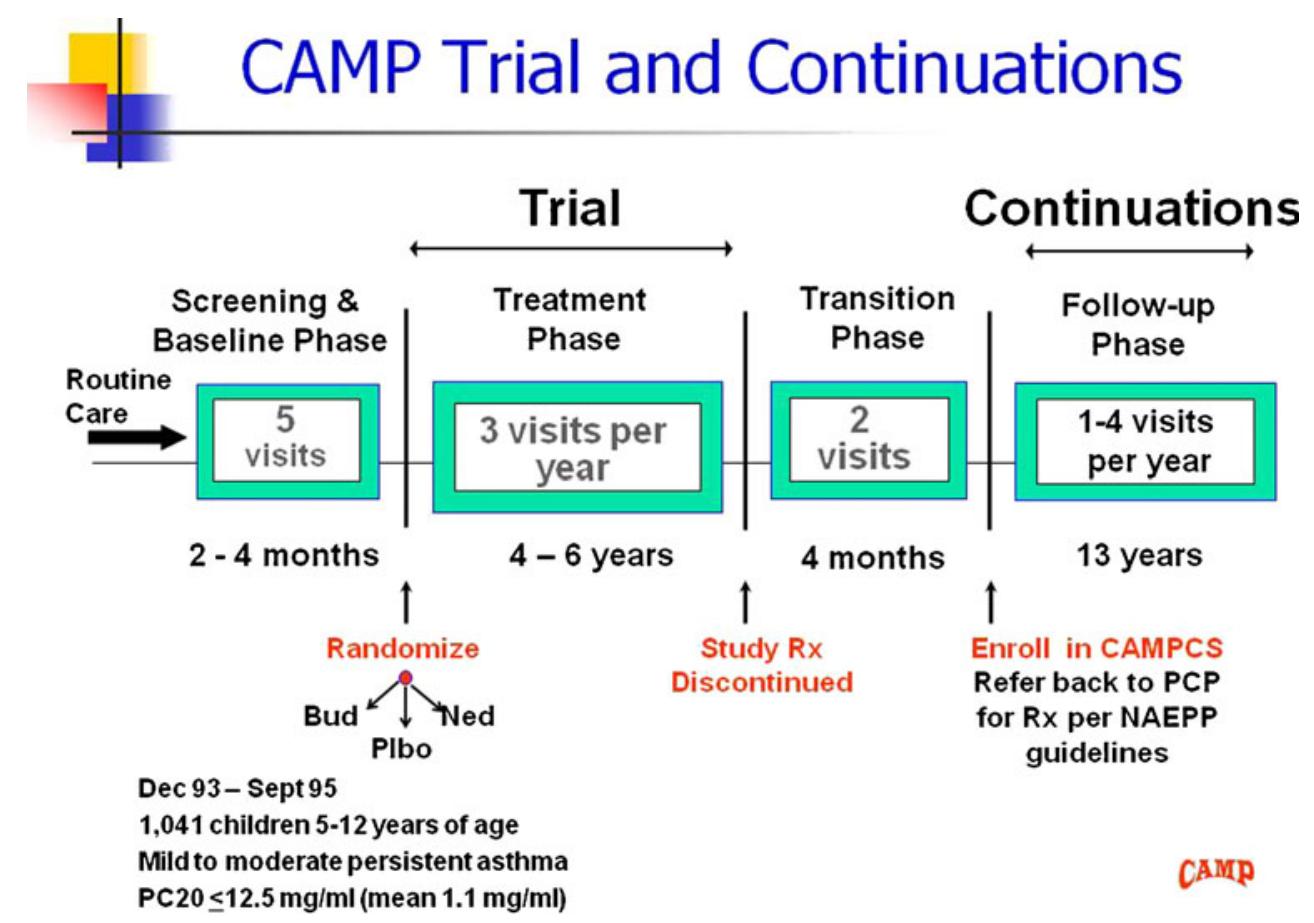

height (mean $-1.1 \mathrm{~cm}$ ) compared with placebo at the end of the trial. The budesonide children were predicted to reach the same mean adult height as the placebo and nedocromil groups on the basis of their growth velocity and bone age at the end of the CAMP trial; however, the budesonide group actually maintained significantly reduced height $(-0.9 \mathrm{~cm}$; $P=0.01)$ compared with the placebo group after an additional 4.8 years for a total follow-up of 9.1 years [5••].

BMDs measured by dual-energy X-ray absorptiometry (DEXA) scans of the lumbar spine were obtained at baseline and at least one follow-up BMD at a median duration of just over seven years from 877 (93\%) of the children in both the treatment and follow-up phases of CAMP [7]. The relationships between bone mineral accretion $\left(\mathrm{g} \mathrm{cm}^{-2}\right.$ year $\left.^{-1}\right)$ and both cumulative number of oral corticosteroids (prednisone) bursts and cumulative ICS dose converted to budesonide equivalents were assessed. A dose-dependent ( $\geq 5$ courses over a median of seven years; $P=0.0002$ ) decrease in annual bone mineral accretion in boys but not girls from oral corticosteroids was found that resulted in an increased risk of osteopenia. More recently, the reduced bone mineral accretion was seen to only occur in the boys with vitamin D insufficiency, thus providing a possible means of preventing this effect of oral corticosteroids [8•]. There was a small decrease in bone mineral accretion in boys only with cumulative ICS dose that was not associated with a risk of osteopenia. No increased risk of fractures was found for either oral corticosteroids or ICSs.

The ICSs are well known to induce dose-dependent suppression of the HPA axis and in very high doses have been associated with Cushing's syndrome and adrenal insufficiency.
Although low to medium doses of short-term ICS have been generally found to be devoid of significant adrenal suppression, with prolonged use it was unknown whether there might be a long-term cumulative effect on the HPA axis with lower doses. HPA axis was assessed in an ancillary study of 63 children entering the CAMP trial at baseline, 12 , and 36 months by cortisol response to $0.25 \mathrm{mg}$ cosyntropin and 24-hour urinary free cortisol measurement [9]. No significant effect of $400 \mu \mathrm{g} \mathrm{day}^{-1}$ budesonide over the three years was found, despite sufficient systemic activity to produce growth suppression.

At the end of the initial CAMP trial all patients were assessed for cataracts by lens photography [2]. One patient had a small opacity that was diagnosed by slit lamp examination as a barely measurable $(<5 \mathrm{~mm})$ PSC. At $12-13$ years after initial randomization, all CAMP participants were encouraged to have slit lamp examinations for cataracts that were funded by CAMP [10]. Of the original cohort 232 patients $(22.1 \%)$ received slit lamp examinations. There were 16 patients with cataract findings, but no significant difference in the percentage of patients who received corticosteroids or never received corticosteroids over CAMP and follow-up (6.7\% versus $8.3 \%$, respectively), nor was there an effect of oral corticosteroids on the development of cataracts.

\section{Phenotypes of childhood asthma}

Post-hoc analysis of the CAMP cohort revealed different asthma phenotypes on the basis of physiologic and clinical characteristics. A subset of children had progressive 
reduction in lung function over time, irrespective of treatment [11]. A loss of at least $1 \%$ predicted per year in post and pre-bronchodilator $\mathrm{FEV}_{1}$ was detected in $26 \%$ and $19 \%$, respectively, of the cohort over four to six years of the treatment phase. Those who had progressive loss of lung function were likely to be male, younger age, with a higher post-bronchodilator $\mathrm{FEV}_{1} \%$ predicted at baseline.

BDR upon entry into CAMP was found to be a powerful, if not the only phenotypic predictor of lung function response to therapy with ICS over the four years of the treatment trial. Increased BDR at baseline was associated with improvements in lung function over time, a higher pre-bronchodilator $\mathrm{FEV}_{1}$ and $\mathrm{FEV}_{1} / \mathrm{FVC}$ ratio and $\mathrm{FVC}$ at four years [12]. Fifty-two CAMP participants had a persistently increased BDR $(>12 \%$ improvement in $\mathrm{FEV}_{1}$ at each visit) over the four-year trial; and this group seemed to define a particular phenotype with different outcomes from those with an increased BDR at baseline alone. Persistent BDR was associated with a lower $\mathrm{FEV}_{1}$ at the end of study trial. Those with persistent BDR over just three visits had similar outcomes [12]. Participants with persistent BDR had an increased frequency of asthma exacerbation, increased nocturnal awakening, and increased school absence. For participants with persistent BDR, increased AHR was a predictor of persistence, suggesting that persistent BDR may be a clinical manifestation of AHR [12]. Factors associated with a persistent BDR included a lower baseline prebronchodilator $\mathrm{FEV}_{1}$, higher $\log \mathrm{IgE}$, and lack of ICS therapy [12].

Some participants during the treatment phase had persistent symptoms (on the basis of diary card recording of at least " 1 " for a mean of $\geq 2$ days per week of symptoms for four weeks within a four-month period) and/or severe exacerbation (use of $\geq 3$ days oral steroid or need for emergency department (ED) visit or hospitalization for asthma) [13•]. Only $26 \%$ of the ICS-treated group compared with $37 \%$ in the nedocromil group and $44 \%$ in the placebo groups $(P<0.0001)$ had persistent symptoms. Similarly, $21 \%, 30 \%$, and $37 \%$ in the ICS, nedocromil, and placebo groups, respectively, had severe exacerbation. Participants with persistent symptoms were more likely not to have been treated with the ICS, and have lower $\mathrm{FEV}_{1}$ / forced vital capacity (FVC) ratio and greater AHR. Participants with severe exacerbation were younger, with a history of previous exacerbation, and also had lower $\mathrm{FEV}_{1}$ / FVC ratio, greater AHR, and higher peripheral eosinophil count at baseline. Although the presence of persistent asthma symptoms was significantly associated with having severe asthma exacerbation, a subpopulation of subjects $(14 \%)$ with no documented persistent symptoms had one or more instances of severe exacerbation.

Participants with mild to moderate asthma do not all behave in the same way. Some develop deterioration in lung function whereas a few manifest with persistent BDR; some have persistent symptoms whereas asthma in some is characterized by the occurrence of severe exacerbation. Hence, assessment of impairment and risk features of asthma should be performed separately.

\section{Predictors of response to inhaled corticosteroid}

CAMP also provided data regarding predictors of poor ICS response. In-utero smoke exposure was associated with blunting of the beneficial effects of ICS therapy on AHR [14•]. Overweight or obese children had a decreased response to ICSs in both measures of lung function and asthma exacerbation compared with children of normal weight [15•]. Poor lung function response (lack of at least a $7.5 \%$ improvement in pre-bronchodilator $\mathrm{FEV}_{1}$ at any of the study visits) was found in approximately $24 \%$ of the 311 participants randomized to the ICS treatment arm [16*0]. Poor responders were likely to be younger, and had higher pre-bronchodilator FEV 1 $\%$ predicted, lower BDR to albuterol, and lower AHR. Multivariable analysis showed only BDR and the two single nucleotide polymorphisms (SNPs), FCER2 and CRHR1, as predictors of poor ICS response. Reduced AHR at baseline was the best predictor of improvement of patients who were not placed on ICS therapy. The differences in the prediction of treatment response between baseline BDR and AHR suggest that these two measures are independent predictors of treatment response [17].

\section{Natural history of childhood asthma}

\section{Remission of asthma}

Prevalence of remitting asthma varies in different epidemiologic studies. In general, the course of asthma in children gets better over time with reduced exacerbation and reported symptoms and improving AHR; our data, however, revealed that fewer than $10 \%$ are likely to be in remission during adolescence, quite different from most published data [5••, $18 \bullet \cdot$. Those who are likely to have remitting asthma in adolescence are those who are not sensitized and exposed to relevant indoor aeroallergens, with milder disease features in early childhood, older age at presentation, less AHR, and better lung function.

Lung function

Inhaled anti-inflammatory therapy did not protect against a progressive deterioration of lung function [11, 19]. Distinct patterns in lung growth (using annual sex-age-specific mean values of pre-bronchodilator $\mathrm{FEV}_{1}, \mathrm{FVC}, \mathrm{FEV}_{1} /$ $\mathrm{FVC} \%$ and growth velocity) were seen in children in 
CAMP using longitudinal measures during the treatment phase and four years of observational phase, compared with children without asthma [19]. No differences in these patterns were detected between the two active treatment groups compared with placebo. The comparative group were 5,415 children without a doctor diagnosis or self and parent report of wheeze or asthma, enrolled as first or second graders in the cohort from schools in six cities in the US between 1974 and 1979 (H6CS).

Differences in $\mathrm{FEV}_{1}, \mathrm{FVC}$, and $\mathrm{FEV}_{1} / \mathrm{FVC}$ trends over time between asthmatics and controls were found. Using lung growth velocities, $\mathrm{FEV}_{1}$ for both boys and girls in the two cohorts track similarly until age 10 years when the growth velocity in children with asthma starts to lag. Children with asthma had higher FVC velocities until peak growth velocities were achieved at age 14 years for boys (0.56 $\mathrm{L} \mathrm{y}^{-1}$ for CAMP and $0.53 \mathrm{~L} \mathrm{y}^{-1}$ for H6CS) and at age 12 years for girls $\left(0.36 \mathrm{~L} \mathrm{y}^{-1}\right.$ for CAMP and $0.33 \mathrm{~L} \mathrm{y}^{-1}$ for H6CS). After age 15 years for boys and 13 years for girls, annual velocities are similar. The percentage of children with asthma whose $\mathrm{FEV}_{1}$ fell below the lower limit of normal (LLN: $<5 \%$ predicted percentile using the sexrace-specific prediction equations) was $12 \%$ at age six years and $19 \%$ at age 18 in boys. The difference was smaller in girls with $11 \%$ of girls in CAMP having a $\mathrm{FEV}_{1}$ below the LLN at age six, $16 \%$ at age 14 , and only $13 \%$ at age 18 . The presence of airflow limitation among asthmatics was magnified by use of the $\mathrm{FEV}_{1} / \mathrm{FVC}$ ratio. Thirty one percent of boys had $\mathrm{FEV}_{1} / \mathrm{FVC}$ ratio below the LLN at age six and $52 \%$ were below the LLN at age $18 ; \mathrm{FEV}_{1} / \mathrm{FVC}$ ratio in $31 \%$ of girls was below the LLN at age seven years and in $51 \%$, it was below the LLN at age 18 years [19].

During the CAMP trial, a decrease in AHR occurred in both boys and girls from age 5 to 11 years. This decrease continued in boys after age 11, but plateaued in girls, without any further decrease from ages 11 to 18 years [20].

\section{Exacerbation of asthma}

The exclusion of children with two or more hospitalizations within the year before CAMP was required to maximize patient safety and exclude patients with more severe and uncontrolled disease. At enrollment, $31 \%(n=320)$ of CAMP participants reported being hospitalized for asthma at least once during their lives, with only $6 \%(n=66)$ of participants having been hospitalized once during the year before the trial. CAMP participants with previous hospitalization for asthma had more severe asthma defined by airway obstruction (lower $\mathrm{FEV}_{1} / \mathrm{FVC}$ ratio), and a younger age of asthma onset and longer duration of the disease. Their families also had fewer psychological resources so may have had a greater difficulty effectively managing the child's illness well enough to avoid hospitalization [21].
A prospective evaluation of the association between $\mathrm{FEV}_{1} \%$ predicted, symptoms, and health care utilization revealed a clear relationship between pre-bronchodilator $\mathrm{FEV}_{1} \%$ and future risk of exacerbation. Compared with children with an $\mathrm{FEV}_{1} \geq 100 \%$ predicted, children with $\mathrm{FEV}_{1} 80$ to $99 \%, 60$ to $79 \%$, and $\leq 60 \%$ were $1.3,1.8$, and 4.8 , respectively, more likely to have a serious asthma exacerbation during the ensuing four months [22].

Traditionally, measurements of airway function, symptoms and/or activity limitation, medication use, inflammatory and atopic markers, and morbidity are used in the assessment of asthma. However, correlations between measurements of asthma health status, the overall burden, or impairment of health related to asthma, are weak and inconsistent, suggesting these outcomes actually comprise independent components of asthma health status. Using factor analysis and incorporating the extensive clinical data collected as part of the CAMP trial enabled examination of the major domains previously identified for asthma and the consistency of the factors identified across time and treatment arm. In each of the study windows, factor analysis consistently identified five factors that explained between $50 \%$ and $60 \%$ of the common variance suggesting that each of these factors provides independent information for assessment of asthma. Factors identified included:

1. inflammatory markers;

2. symptoms and/or medication use;

3. asthma exacerbation

and measures of lung function, subdivided into:

4. FEV $\mathrm{FV}_{1}$ and FVC; and

5. BDR and the $\mathrm{FEV}_{1} / \mathrm{FVC}$ ratio.

Exploratory analysis suggests that fractional exhaled nitric oxide loaded with the atopy and/or inflammatory factor, and sputum eosinophil measurements separated out as a sixth, factor [23].

Building on this analysis, Forno et al. developed and validated a predictive score for asthma exacerbation [24•]. The authors used an exploratory dataset from an asthma population in Costa Rica to identify a similar cluster of variables corresponding to six categories: asthma symptoms, health-care utilization, medical history, asthma medications, laboratory tests, and AHR; the categories laboratory tests and AHR were, however, excluded for ease of use of the clinical score in the office setting. The resulting score with 17 total points was grouped into three categories: low risk (score, $\leq 5$ ), intermediate or average risk (score, 6-8), and high risk (score, $\geq 9$ ). Using the CAMP asthma population as a validation dataset, the hazard ratios for an exacerbation after one-year follow-up in the low-risk and high-risk groups were 0.6 (95\% CI, 0.5-0.7) and 1.9 (95\% CI, 1.4 $2.4)$, respectively, with similar results at two years. 
As indicated earlier, the demographic and laboratory predictors of having persistent symptoms were not identical to the predictors of severe asthma exacerbation [13•]. In a second analysis, investigators examined 17 phenotypic variables together with polymorphisms in FCER2 and CRHR1. A history of previous asthma exacerbation, younger age, and higher IgE level were associated with recurrent exacerbation. Variables that predicted risk of recurrent asthma exacerbation were not the same as predictors of impairment [10]. This supports the current focus of the National Asthma Education and Prevention Program guidelines on the two separate domains of asthma control - impairment and risk [3].

Vitamin D has been shown to have effects on innate and adaptive immunity that could modify the severity of asthma exacerbation. Published together, work by Poon et al. [25] and the genetics ancillary group [26], were the first reports linking vitamin $\mathrm{D}$ and asthma. Brehm et al. further examined the relationship between vitamin D levels and risk of hospitalization or ED visits in a prospective manner in the CAMP study. Thirty-five percent of all subjects were categorized as vitamin D insufficient $\left(<30 \mathrm{ng} \mathrm{mL}^{-1}\right)[27 \bullet]$. Mean vitamin D levels were lowest for African-American subjects and highest for white subjects. After adjusting for age, sex, body mass index, income, and treatment group, insufficient vitamin D status was associated with higher odds of severe exacerbation over a four-year period (OR, $1.5 ; 95 \%$ CI, $1.1-1.9 ; P=0.01$ ).

In CAMP, children with a history of severe asthma exacerbation requiring hospital admission started having symptoms early and eventually developed greater airflow limitation. Whether the impairment in lung function was a result of an early injury or low lung function preceded the risk of exacerbation in the first place could not be ascertained. From the CAMP data, having low lung function at baseline predisposed to a greater risk of future exacerbation. Although the presence of exacerbation is a marker of asthma severity, there are patients who remain exacerbation prone without associated persistent symptoms or even lung function impairment. Therefore, a comprehensive assessment of impairment and risk should be undertaken and therapy designed for specific aspects of asthma control should be identified. Vitamin D supplementation in children whose level is insufficient seems a promising intervention to reduce asthma exacerbation.

\section{Pharmacogenetics of asthma}

Investigators in the Channing Laboratory, Brigham and Women's Hospital, developed the CAMP genetics ancillary study group combining the extensive phenotype information obtained from the CAMP participants. Use of an integrative genomic approach to map regulatory variants for more than 1,500 genes revealed that genetic variants are responsible for inter-individual differences in gene expression and contribute to disease susceptibility [28]. In a genome-wide expression study of peripheral blood CD4 $+\mathrm{T}$ cells, Hunninghake and colleagues observed a relationship between expression of the interleukin 17 receptor B (IL17R $\beta$ ) and variation in total serum IgE level in asthmatics. Interestingly, sex-stratified analysis revealed that the correlation between IL17R $\beta$ and $\operatorname{IgE}$ was restricted to males only, providing evidence of the importance of sex in genomic regulation of IgE [29]. CAMP has made significant contributions to the mapping of susceptibility genes. In genomewide association studies, the CAMP cohort has been studied to map multiple asthma-susceptibility genes, including PDE4D [30], IL1RL1, TSLP, and IL33 [31]. In addition, a functional variant in MMP12 was found to confer a positive effect on lung function in children with asthma and associated with a reduced risk of COPD in adult smokers [32••]. An additional focus of the CAMP genetic ancillary group has been the identification of novel pharmacogenetic determinants of asthma therapy. Genetic variation in FCER 2 may help predict ICS response in asthma. Tantisira et al. demonstrated an association between variation in the low-affinity IgE receptor gene, FCER2, IgE level, and severe exacerbation in patients with asthma on ICS [33]. Second, a functional variant $T B X 21$, which encodes for T-bet, is associated with improved FEV $_{1}$ response to ICSs [34], and polymorphisms of the corticotrophin-releasing hormone receptor (CRHRl) are associated with improved lung function in asthmatics treated with ICSs [35]. In a fourth analysis, a functional variant in a novel pharmacogenetic gene, the glucocorticoid-induced transcript 1 gene (GLCCI1), was shown to be associated with decrements in the response to ICS in patients with asthma, results that were replicated in four independent populations. The identified variant allele has a frequency approaching 0.4 , suggesting approximately $16 \%$ of the population will be homozygous for the genotype responsible for the more limited response to ICS [36••]. Parallel to the work revealing the effects of genetics on response to ICSs, Litonjua et al. published the first major description of a replicable gene that is not the $\beta 2$-adrenergic receptor, but is associated with $\beta$-agonist response, identifying the arginase 1 gene $(A R G l)$ as a potential $\beta$-agonist response gene in both children and adults with asthma [37]. Himes et al. analyzed asthmatic subjects from six independent clinical trials for association with BDR, with replication in two independent asthma cohorts, including the CAMP population [38]. A strong association was noted for a SNP on chromosome 2 near SPATS2L. Inhibition of SPATS2L expression is associated with increased $\beta 2$-adrenergic receptor levels, suggesting that SPATS2L may be an important regulator of $\beta 2$-adrenergic receptor down-regulation. These results provide important evidence that genetic variants may be useful in predicting therapeutic response in asthma. 


\section{Conclusion}

In conclusion, CAMP investigations have established that long-term use of ICS at a low-medium dose over 4-6 years is safe in children although it does produce a small decrement in height that is not progressive after the first few years. Although ICS reduce the risk of exacerbation, and improve symptoms and baseline lung function overall, these effects disappear upon discontinuation of therapy. In addition, ICS therapy does not prevent the reduction in lung function seen in a number of patients nor does it seem to affect the natural history of childhood asthma. Various phenotypic features and genotypic variants modify both long-term asthma outcomes and response to ICS in childhood asthma.

Disclosure R. Covar: none; A. Fuhlbrigge: board membership (Merck) and consultant (Merck, GlaxoSmithKline, ICON Medical Imaging, Sunovion, Lovelace Respiratory Research Institute); P. Williams: speakers' bureau (GlaxoSmithKline); and H.W. Kelly: consultant (GlaxoSmithKline, Novartis, Merck, AstraZeneca).

\section{References}

Papers of particular interest, published recently, have been highlighted as:

- Of importance

•- Of major importance

1. Childhood Asthma Management Program Research Group. NHLBI Division of Lung Diseases. The Childhood Asthma Management Program (CAMP): design, rationale, and methods. Controlled Clin Trials. 1999;20:91-120.

2. Childhood Asthma Management Program Research Group. Longterm effects of budesonide or nedocromil in children with asthma. N Engl J Med. 2000;343:1054-63.

3. Expert Panel Report 3 (EPR-3): Guidelines for the Diagnosis and Management of Asthma-Summary Report 2007. J Allergy Clin Immunol. 2007;120:S94-138

4. Strunk RC, for the Childhood Asthma Management Program Research Group. Childhood Asthma Management Program: Lessons learned. J Allergy Clin Immunol. 2007;119:36-42.

5. •- Strunk RC, Sternberg AL, Szefler SJ, et al. Long-term budesonide or nedocromil treatment, once discontinued, does not alter the course of mild to moderate asthma in children and adolescents. J Pediatr. 2009;154:682-7. The follow up of the CAMP cohort suggests that the beneficial effects of the therapy are not sustained after discontinuation of long-term anti-inflammatory medication. The initial effect of ICS on growth is still evident. For all treatment groups, reductions in measures of asthma morbidity were found.

6. Kelly HW. Potential adverse effects of the inhaled corticosteroids. J Allergy Clin Immunol. 2003;112:469-78.

7. Kelly HW, Van Natta ML, Covar RA, for the Childhood Asthma Management Program Research Group, et al. Effect of long-term corticosteroid use on bone mineral density in children: A prospective longitudinal assessment in the Childhood Asthma Management Program study. Pediatrics. 2008;122:e53-61.

8. - Tse SM, Kelly HW, Litonjua AA, for the Childhood Asthma Management Program Research Group, et al. Corticosteroid use and bone mineral accretion in children with asthma: Effect modification by vitamin D. J Allergy Clin Immunol. 2012;130:53-60. Serum vitamin $D$ levels modify the effects of corticosteroids on bone growth, primarily in boys. Bone mineral accretion is compromised with increased use of systemic corticosteroids only in vitamin D-deficient boys. .

9. Bacharier LB, Raissy HH, Wilson L, et al. The long-term effect of budesonide on hypothalamic-pituitary-adrenal axis function in children with mild to moderate asthma. Pediatrics. 2004;113:1693-9.

10. Raissy HH, Sternberg AL, Williams P, for the Childhood Asthma Management Program Research Group, et al. Risk of cataracts in the Childhood Asthma Management Program Cohort. J Allergy Clin Immunol. 2010;126:389-92.

11. Covar RA, Spahn JD, Murphy JR, Szefler SJ, for the Childhood Asthma Management Program Research Group. Progression of asthma measured by lung function in the Childhood Asthma Management Program. Am J Respir Crit Care Med. 2004;170:234-41.

12. Sharma S, Litonjua AA, Tantisira KG, for the Childhood Asthma Management Program, et al. Clinical predictors and outcomes of consistent bronchodilator response in the childhood asthma management program. J Allergy Clin Immunol. 2008;122:921-8.

13. - Wu AC, Tantisira K, Li L, Schuemann B, for the Childhood Asthma Management Program, et al. Predictors of symptoms are different from predictors of severe exacerbations from asthma in children. Chest. 2011;140:100-7. Comparison of children with persistent symptoms and those with severe exacerbations using analysis of diary cards. Predictors of persistent symptoms included non-treatment with ICS, airway obstruction, and increased AHR. Predictors of exacerbations included younger age, previous hospitalization, or ED visit in the previous year, and oral steroid use in the previous three months.

14. • Cohen RT, Raby BA, Van Steen K, et al. In utero smoke exposure and impaired response to inhaled corticosteroids in children with asthma. J Allergy Clin Immunol. 2010;126:491-7. Early life exposure, such as in utero smoke, can affect AHR over time and response to asthma therapy. Long-term effect of this exposure cannot be ignored.

15. - Forno E, Lescher R, Strunk R, for the Childhood Asthma Management Program, et al. Decreased response to inhaled steroids in overweight and obese asthmatic children. J Allergy Clin Immunol. 2011;127:741-9. Effect of ICS therapy, as measured by lung function and clinical outcomes, is reduced in overweight or obese children compared with children of normal weight. Perhaps these children may require higher doses or alternative medications to achieve optimum asthma control.

16. •- Rogers AJ, Tantisira KG, Fuhlbrigge AL, for the Childhood Asthma Management Program Research Group, et al. Predictors of poor response during asthma therapy differ with definition of outcome. Pharmacogenetics. 2009;10:1231-42. This paper, analyzing phenotypic variables and key genetic polymorphisms, noted significant differences in predictors of exacerbation vs. lung function response. This has implications for future study design.

17. Tantisira KG, Fuhlbrigge AL, Tonascia J, for the Childhood Asthma Management Program Research Group, et al. Bronchodilation and bronchoconstriction: Predictors of future lung function in childhood asthma. J Allergy Clin Immunol. 2006;117:1264-71.

18. • Covar R, Strunk R, Zeiger RS, et al. Predictors of remitting, periodic, and persistent childhood asthma. J Allergy Clin Immunol. 2010;125:359-66. The belief that children with mild asthma are likely to outgrow their disease is not supported by the findings from the CAMP cohort. Fewer than $10 \%$ of children with a history of mild to moderate asthma at school age have no active asthma in adolescence. Those with remitting asthma are likely not to be sensitized and exposed to relevant indoor allergens and have milder disease features in early childhood.

19. Strunk RC, Weiss ST, Yates KP, for the Childhood Asthma Management Program Research Group, et al. Mild to moderate asthma affects lung growth in children and adolescents. J Allergy Clin Immunol. 2006;118:1040-7. 
20. Tantisira KG, Colvin R, Tonascia J, for the Childhood Asthma Management Program Research Group, et al. Airway responsiveness in mild to moderate childhood asthma; Sex influences on the natural history. Am J Respir Crit Care Med. 2008; 178:325-31.

21. Bacharier LB, Dawson C, Bloomberg GR, for the Childhood Asthma Management Program, et al. Hospitalization for asthma: Atopic, pulmonary function, and psychological correlates among participants in the Childhood Asthma Management Program. Pediatrics. 2003;112:e85-92.

22. Fuhlbrigge AL, Weiss ST, Kuntz KM, Paltiel AD, for the CAMP Research Group. Forced expiratory volume in 1 second percentage improves the classification of severity among children with asthma. Pediatrics. 2006;118:e347-55.

23. Holt EW, Cook EF, Covar RA, Spahn J, Fuhlbrigge AL. Identifying the components of asthma health status in children with mild to moderate asthma. J Allergy Clin Immunol. 2008; 121:1175-80.

24. - Forno E, Fuhlbrigge A, Soto-Quirós ME, et al. Risk factors and predictive clinical scores for asthma exacerbations in childhood. Chest. 2010;138:1156-65. An asthma exacerbation clinical score for primary care clinicians to identify children at risk for exacerbation, initially evaluated in a sample of Costa Rican children, is shown to have high predictive value in the CAMP cohort. The scoring system incorporates reported asthma symptoms, current medications, history of health care utilization, personal/family history of atopy, and smoke exposure.

25. Poon AH, Laprise C, Lemire M, et al. Association of vitamin D receptor genetic variants with susceptibility to asthma and atopy. Am J Respir Crit Care Med. 2004;170:967-73.

26. Raby BA, Lazarus R, Silverman EK, et al. Association of Vitamin $\mathrm{D}$ receptor gene polymorphisms with childhood and adult asthma. Am J Respir Crit Care Med. 2004;170:1057-65.

27. • Brehm JM, Schuemann B, Fuhlbrigge AL, for the Childhood Asthma Management Program Research Group, et al. Serum vitamin D levels and severe asthma exacerbations in the Childhood Asthma Management Program study. J Allergy Clin Immunol. 2010;126:52-8. Baseline vitamin D insufficiency is associated with a higher risk of emergency room visits and hospitalization during the trial, irrespective of active treatment.
28. Murphy A, Chu J-H, Xu M, et al. Mapping of numerous diseaseassociated expression polymorphisms in primary peripheral blood CD4+ lymphocytes. Hum Mol Genet. 2012;19:4745-57.

29. Hunninghake GM, Chu J-H, Sharma SS, et al. The CD4+ T-cell transcriptome and serum IgE in asthma: IL17RB and the role of sex. BMC Pulmonary Medicine. 2011;11:17.

30. Himes BE, Hunninghake GM, Baurley JW, et al. Genome-wide association analysis identifies $P D E 4 D$ as an asthma-susceptibility gene. Am J Hum Genet. 2009;84:581-93.

31. Torgerson DG, Ampleford EJ, Chiu GY, et al. Meta-analysis of genome-wide association studies of asthma in ethnically diverse North American populations. Nat Genet. 2011;43:887-92.

32. •• Hunninghake GM, Cho MH, Tesfaigzi Y, et al. MMP12, lung function, and COPD in high-risk populations. N Engl J Med. 2009;361:2599-608. This paper reported that a variant in MMP12 is important in lung function in asthma and COPD.

33. Tantisira KG, Silverman ES, Mariani TJ, et al. FCER2: A pharmacogenetic basis for severe exacerbations in children with asthma. J Allergy Clin Immunol. 2007;120:1285-91.

34. Tantisira KG, Hwang ES, Raby BA, et al. TBX21: A functional variant predicts improvement in asthma with the use of inhaled corticosteroids. Proc Natl Acad Sci. 2004;101:18099-104.

35. Tantisira KG, Lake S, Silverman ES, et al. Corticosteroid pharmacogenetics: association of sequence variants in CRHRI with improved lung function in asthmatics treated with inhaled corticosteroids. Hum Mol Genet. 2004;13:1353-9.

36. •• Tantisira KG, Lasky-Su J, Harada M, et al. Genomewide association between GLCCI1 and response to glucocorticoid therapy in asthma. N Engl J Med. 2011;365:1173-83. The paper reveals that a functional GLCCI1 variant is associated with substantial decrements in the response to inhaled corticosteroids in patients with asthma, demonstrating that genetic variants may be useful in predicting therapeutic response in asthma.

37. Litonjua AA, Lasky-Su J, Schneiter K, et al. ARG1 is a novel bronchodilator response gene. Screen and replication in four asthma cohorts. Am J Respir Crit Care Med. 2008;178:688-94.

38. Himes BE, Jiang X, Hu R, Wu AC, et al. Genome-Wide Association Analysis in Asthma Subjects Identifies SPATS2L as a Novel Bronchodilator Response Gene. Plos Genetics PLoS Genet 8(7): e1002824. doi:10.1371/journal.pgen.1002824 